\title{
Infinite operator-sum representation of density operator for a dissipative cavity with Kerr medium derived by virtue of entangled state representation
}

\author{
Li-yun Hu*and Hong-yi Fan \\ Department of Physics, Shanghai Jiao Tong University, Shanghai, 200030, China
}

October 30, 2018

\begin{abstract}
By using the thermo entangled state representation we solve the master equation for a dissipative cavity with Kerr medium to obtain density operators' infinite operator-sum representation $\rho(t)=\sum_{m, n, l=0}^{\infty} M_{m, n, l} \rho_{0} \mathcal{M}_{m, n, l}^{\dagger}$. It is noticable that $M_{m, n, l}$ is not hermite conjugate to $\mathcal{M}_{m, n, l}^{\dagger}$, nevertheless the normalization $\sum_{m, n, l=0}^{\infty} \mathcal{M}_{n m, l}^{\dagger} M_{m, n, l}=1$ still holds, i.e., they are trace-preserving in a general sense. This example may stimulate further studiing if general superoperator theory needs modification.
\end{abstract}

\section{Introduction}

For an open quantum system interacting with the enviroment, one uses superoperators to describe the evolution of density operator from an initial $\rho_{0}$ (pure state or mixed state) into a final state $\rho$. A superoperator plays a role of linear map from $\rho_{0} \rightarrow \rho$, which has an operator-sum representation [1, 2]

$$
\rho=\sum_{n} M_{n} \rho_{0} M_{n}^{\dagger}
$$

where the operators $M_{n}$ and $M_{n}^{\dagger}$ are usually hermite conjugate to each other, and obey the normalization condition [3],

$$
\sum_{n} M_{n}^{\dagger} M_{n}=1 .
$$

$M_{n}$ is named Kraus operator [4, 5, $M_{n}$ and $M_{n}^{\dagger}$ are hermite conjugate to each other. Such an operator-sum representation is the core of POVM (positive operator-valued measure).

In our very recent papers [6, 17, based on the thermo entangled state representation, we have derived some density operators that are in infinite dimensional operator-sum forms, for example, those for describing amplitude-damping channel and laser process, the corresponding Kraus operators are also obtained and their normalization proved, which implies trace-preserving.

Then an important and interesting question challenges us: is there an infinite operator-sum representation of density operator in the infinite sum form $\rho(t)=\sum_{n=0}^{\infty} M_{n} \rho_{0} \mathcal{M}_{n}^{\dagger}$, where the normalization $\sum_{n=0}^{\infty} \mathcal{M}_{n}^{\dagger} M_{n}=1$ still holds, but $M_{n}$ and $\mathcal{M}_{n}^{\dagger}$ are not hermite conjugate to each

*Corresponding author. Address: Department of Physics, Shanghai Jiao Tong University, Shanghai, 200030, China. Tel./fax: +86 2162932080. E-mail addresses: hlyun2008@126.com, hlyun@sjtu.edu.cn (L.-y. $\mathrm{Hu})$. 
other? The answer is affirmative. In this paper, by using the thermo entangled state representation we solve the master equation for a dissipative cavity with Kerr medium to obtain its density operator, and find its infinite operator-sum representation really possesses such a "strange structure", this may bring attention of theoreticians who might wonder if the general theory of superoperators should be modified.

\section{Brief review of the thermo entangled state representation}

In this section, we briefly review the thermo entangled state representation (TESR). Enlightened by the Thermo Dynamic Theory of Takahashi-Umezawa [8, 9, 10, we have constructed the TESR in doubled Fock space [1],

$$
|\eta\rangle=\exp \left(-\frac{1}{2}|\eta|^{2}+\eta a^{\dagger}-\eta^{*} \tilde{a}^{\dagger}+a^{\dagger} \tilde{a}^{\dagger}\right)|0, \tilde{0}\rangle
$$

in which $\tilde{a}^{\dagger}$ is a fictitious mode accompanying the real photon creation operator $a^{\dagger},|0, \tilde{0}\rangle=|0\rangle|\tilde{0}\rangle$, $|\tilde{0}\rangle$ is annihilated by $\tilde{a},\left[\tilde{a}, \tilde{a}^{\dagger}\right]=1$. Operating $a$ and $\tilde{a}$ on $|\eta\rangle$ in Eq.(3) we obtain the eigen-equations of $|\eta\rangle$,

$$
\begin{aligned}
& \left(a-\tilde{a}^{\dagger}\right)|\eta\rangle=\eta|\eta\rangle, \quad\left(a^{\dagger}-\tilde{a}\right)|\eta\rangle=\eta^{*}|\eta\rangle, \\
& \langle\eta|\left(a^{\dagger}-\tilde{a}\right)=\eta^{*}\langle\eta|, \quad\langle\eta|\left(a-\tilde{a}^{\dagger}\right)=\eta\langle\eta| .
\end{aligned}
$$

Note that $\left[\left(a-\tilde{a}^{\dagger}\right),\left(a^{\dagger}-\tilde{a}\right)\right]=0$, thus $|\eta\rangle$ is the common eigenvector of $\left(a-\tilde{a}^{\dagger}\right)$ and $\left(\tilde{a}-a^{\dagger}\right)$.

Using the normally ordered form of vacuum projector $|0, \tilde{0}\rangle\langle 0, \tilde{0}|=: \exp \left(-a^{\dagger} a-\tilde{a}^{\dagger} \tilde{a}\right):$, and the technique of integration within an ordered product (IWOP) of operators [12, 13], we can easily prove that $|\eta\rangle$ is complete and orthonormal,

$$
\begin{aligned}
\int \frac{d^{2} \eta}{\pi}|\eta\rangle\langle\eta| & =\int \frac{d^{2} \eta}{\pi}: \exp \left(-|\eta|^{2}+\eta a^{\dagger}-\eta^{*} \tilde{a}^{\dagger}+a^{\dagger} \tilde{a}^{\dagger}+\eta^{*} a-\eta \tilde{a}+a \tilde{a}--a^{\dagger} a-\tilde{a}^{\dagger} \tilde{a}\right):=(15) \\
\left\langle\eta^{\prime} \mid \eta\right\rangle & =\pi \delta\left(\eta^{\prime}-\eta\right) \delta\left(\eta^{\prime *}-\eta^{*}\right)
\end{aligned}
$$

The $|\eta=0\rangle$ state

$$
|\eta=0\rangle=e^{a^{\dagger} \tilde{a}^{\dagger}}|0, \tilde{0}\rangle=\sum_{n=0}^{\infty}|n, \tilde{n}\rangle
$$

possesses the properties

$$
\begin{aligned}
a|\eta=0\rangle & =\tilde{a}^{\dagger}|\eta=0\rangle, a^{\dagger}|\eta=0\rangle=\tilde{a}|\eta=0\rangle, \\
\left(a^{\dagger} a\right)^{n}|\eta=0\rangle & =\left(\tilde{a}^{\dagger} \tilde{a}\right)^{n}|\eta=0\rangle .
\end{aligned}
$$

Note that density operators are functions of $\left(a^{\dagger}, a\right)$, i.e., defined in the original Fock space, so they are commutative with operators of $\left(\tilde{a}^{\dagger}, \tilde{a}\right)$ in the tilde space.

\section{Infinite operator-sum representation of density operator for a dissipative cavity with Kerr medium}

In the Markov approximation and interaction picture the master equation for a dissipative cavity with Kerr medium has the form [14, 15, 16.

$$
\frac{d \rho}{d t}=-i \chi\left[\left(a^{\dagger} a\right)^{2}, \rho\right]+\gamma\left(2 a \rho a^{\dagger}-a^{\dagger} a \rho-\rho a^{\dagger} a\right)
$$


where $\gamma$ is decaying parameter of the dissipative cavity, $\chi$ is coupling factor depending on the Kerr medium. Next we will solve the master equation by virtue of the entangled state representation and present the infinite sum representation of density operator.

Operating the both sides of Eq.(10) on the state $|\eta=0\rangle$, letting

$$
|\rho\rangle=\rho|\eta=0\rangle
$$

and using Eq.(8) we have the following state vector equation,

$$
\frac{d}{d t}|\rho\rangle=\left\{-i \chi\left[\left(a^{\dagger} a\right)^{2}-\left(\tilde{a}^{\dagger} \tilde{a}\right)^{2}\right]+\gamma\left(2 a \tilde{a}-a^{\dagger} a-\tilde{a}^{\dagger} \tilde{a}\right)\right\}|\rho\rangle,
$$

the formal solution of $|\rho\rangle$ is

$$
|\rho\rangle=\exp \left\{-i \chi t\left[\left(a^{\dagger} a\right)^{2}-\left(\tilde{a}^{\dagger} \tilde{a}\right)^{2}\right]+\gamma t\left(2 a \tilde{a}-a^{\dagger} a-\tilde{a}^{\dagger} \tilde{a}\right)\right\}\left|\rho_{0}\right\rangle,
$$

where $\left|\rho_{0}\right\rangle=\rho_{0}|\eta=0\rangle, \rho_{0}$ is the initial density operator. By introducing the following operators,

$$
K_{0}=a^{\dagger} a-\tilde{a}^{\dagger} \tilde{a}, \quad K_{z}=\frac{a^{\dagger} a+\tilde{a}^{\dagger} \tilde{a}+1}{2}, \quad K_{-}=a \tilde{a},
$$

and noticing $\left[K_{0}, K_{z}\right]=\left[K_{0}, K_{-}\right]=0$, we can rewrite Eq.(13) as

$$
\begin{aligned}
|\rho\rangle & =\exp \left\{-i \chi t\left[K_{0}\left(2 K_{z}-1\right)\right]+\gamma t\left(2 K_{-}-2 K_{z}+1\right)\right\}\left|\rho_{0}\right\rangle \\
& =\exp \left[i \chi t K_{0}+\gamma t\right] \exp \left\{-2 t\left(\gamma+i \chi K_{0}\right)\left[K_{z}+\frac{-\gamma}{\gamma+i \chi K_{0}} K_{-}\right]\right\}\left|\rho_{0}\right\rangle .
\end{aligned}
$$

With the aid of the operator identity [17]

$$
e^{\lambda(A+\sigma B)}=e^{\lambda A} \exp \left[\sigma B\left(1-e^{-\lambda \tau}\right) / \tau\right]=\exp \left[\sigma B\left(e^{\lambda \tau}-1\right) / \tau\right] e^{\lambda A},
$$

which is valid for $[A, B]=\tau B$, and noticing $\left[K_{z}, K_{-}\right]=-K_{-}$, we can reform Eq.(15) as

$$
|\rho\rangle=\exp \left[i \chi t K_{0}+\gamma t\right] \exp \left[\Gamma_{z} K_{z}\right] \exp \left[\Gamma_{-} K_{-}\right]\left|\rho_{0}\right\rangle,
$$

where

$$
\Gamma_{z}=-2 t\left(\gamma+i \chi K_{0}\right), \Gamma_{-}=\frac{\gamma\left(1-e^{-2 t\left(\gamma+i \chi K_{0}\right)}\right)}{\gamma+i \chi K_{0}} .
$$

In order to deprive of the state $|\eta=0\rangle$ from Eq.(17), using the completeness relation of Fock state in the enlarged space $\sum_{m, n=0}^{\infty}|m, \tilde{n}\rangle\langle m, \tilde{n}|=1$ and noticing $a^{\dagger l}|n\rangle=\sqrt{\frac{(l+n) !}{n !}}|n+l\rangle$, we have

$$
\begin{aligned}
|\rho\rangle & =\exp \left[i \chi t K_{0}+\gamma t\right] \exp \left[\Gamma_{z} K_{z}\right] \sum_{l=0}^{\infty} \frac{\Gamma_{-}^{l}}{l !} a^{l} \rho_{0} a^{\dagger l}|\eta=0\rangle \\
& =\exp \left[i \chi t K_{0}+\gamma t\right] \sum_{l=0}^{\infty} \frac{\Gamma_{-}^{l}}{l !} \exp \left[\Gamma_{z} K_{z}\right] \sum_{m, n=0}^{\infty}|m, \tilde{n}\rangle\left\langle m, \tilde{n}\left|a^{l} \rho_{0} a^{\dagger l}\right| \eta=0\right\rangle \\
& =\sum_{l=0}^{\infty} \frac{\Lambda_{m, n}^{l}}{l !} \exp \left[-i t \chi\left(m^{2}-n^{2}\right)-t \gamma(m+n)\right] \sum_{m, n=0}^{\infty}|m, \tilde{n}\rangle\left\langle m, \tilde{n}\left|a^{l} \rho_{0} a^{\dagger l}\right| \eta=0\right\rangle,
\end{aligned}
$$

where we have set

$$
\Lambda_{m, n} \equiv \frac{\gamma\left(1-e^{-2 t(\gamma+i \chi(m-n))}\right)}{\gamma+i \chi(m-n)}
$$


Further, using

$$
\langle n \mid \eta=0\rangle=|\tilde{n}\rangle, \quad|m, \tilde{n}\rangle=|m\rangle\langle n \mid \eta=0\rangle,
$$

which leads to

$$
\left\langle m, \tilde{n}\left|a^{l} \rho_{0} a^{\dagger l}\right| \eta=0\right\rangle=\langle m|\left\langle\tilde{n}\left|a^{l} \rho_{0} a^{\dagger l}\right| \eta=0\right\rangle=\langle m| a^{l} \rho_{0} a^{\dagger l}(\langle\tilde{n} \mid \eta=0\rangle)=\left\langle m\left|a^{l} \rho_{0} a^{\dagger l}\right| n\right\rangle,
$$

then Eq.(19) becomes

$$
\begin{aligned}
|\rho\rangle & =\sum_{m, n, l=0}^{\infty} \frac{\Lambda_{m, n}^{l}}{l !} \exp \left[-i \chi t\left(m^{2}-n^{2}\right)-\gamma t(m+n)\right]|m, \tilde{n}\rangle\left\langle m\left|a^{l} \rho_{0} a^{\dagger l}\right| n\right\rangle \\
& =\sum_{m, n, l=0}^{\infty} \sqrt{\frac{(n+l) !(m+l) !}{n ! m !}} \frac{\Lambda_{m, n}^{l}}{l !} e^{-i \chi t\left(m^{2}-n^{2}\right)-\gamma t(m+n)}|m, \tilde{n}\rangle \rho_{0, m+l, n+l},
\end{aligned}
$$

where $\rho_{0, m+l, n+l} \equiv\left\langle m+l\left|\rho_{0}\right| n+l\right\rangle$. Using Eq.(21) again, we see

$$
|\rho\rangle=\sum_{m, n, l=0}^{\infty} \sqrt{\frac{(n+l) !(m+l) !}{n ! m !}} \frac{\left(\Lambda_{m, n}\right)^{l}}{l !} e^{-i \chi t\left(m^{2}-n^{2}\right)-\gamma t(m+n)} \rho_{0, m+l, n+l}|m\rangle\langle n \mid \eta=0\rangle .
$$

After depriving $|\eta=0\rangle$ from the both sides of Eq.(24), the solution of master equation (10) appears as infinite operator-sum form

$$
\begin{aligned}
\rho(t) & =\sum_{m, n, l=0}^{\infty} \sqrt{\frac{(n+l) !(m+l) !}{n ! m !}} \frac{\Lambda_{m, n}^{l}}{l !} e^{-i \chi t\left(m^{2}-n^{2}\right)-\gamma t(m+n)}|m\rangle\left\langle m+l\left|\rho_{0}\right| n+l\right\rangle\langle n| \\
& =\sum_{m, n, l=0}^{\infty} \frac{\Lambda_{m, n}^{l}}{l !} e^{-i \chi t\left(m^{2}-n^{2}\right)-\gamma t(m+n)}|m\rangle\left\langle m\left|a^{l} \rho_{0} a^{\dagger l}\right| n\right\rangle\langle n|,
\end{aligned}
$$

Note that the factor $(m-n)$ appears in the denominator of $\Lambda_{m, n}$ (see Eq. (20)), (this is originated from the nonlinear term of $\left.\left(a^{\dagger} a\right)^{2}\right)$, so moving of all $n$-dependent terms to the right of $a^{l} \rho_{0} a^{\dagger l}$ is impossible, nevertheless, we can formally express Eq.(25) as

$$
\rho(t)=\sum_{m, n, l=0}^{\infty} M_{m, n, l} \rho_{0} \mathcal{M}_{m, n, l}^{\dagger}
$$

where the two operators $M_{m, n, l}$ and $\mathcal{M}_{m, n, l}^{\dagger}$ are respectively defined as

$$
\begin{aligned}
M_{m, n, l} & \equiv \sqrt{\frac{\Lambda_{m, n}^{l}}{l !}} e^{-i \chi t m^{2}-\gamma t m}|m\rangle\langle m| a^{l}, \\
\mathcal{M}_{m, n, l}^{\dagger} & \equiv\left\{\sqrt{\frac{\Lambda_{n, m}^{l}}{l !}} e^{-i \chi t n^{2}-\gamma t n}|n\rangle\langle n| a^{l}\right\}^{\dagger},
\end{aligned}
$$

to one's regret, $M_{m, n, l}$ is not hermite conjugate to $\mathcal{M}_{m, n, l}^{\dagger}$. This example may surprise us to wonder if the general superoperator form in Eq. (11) needs modification. 


\section{Further analysis for $\rho(t)$}

Usng the operator identity $e^{\lambda a^{\dagger} a}=: \exp \left[\left(e^{\lambda}-1\right) a^{\dagger} a\right]:$ and the IWOP technique, we can prove that

$$
\begin{aligned}
& \sum_{m, n, l=0}^{\infty} \mathcal{M}_{m, n, l}^{\dagger} M_{m, n, l} \\
= & \sum_{n, l=0}^{\infty} \frac{(n+l) !}{n !} \frac{\left(1-e^{-2 t \gamma}\right)^{l}}{l !} e^{-2 n \gamma t}|n+l\rangle\langle n+l| \\
= & \sum_{n, l=0}^{\infty} \frac{\left(1-e^{-2 t \gamma}\right)^{l}}{l !} a^{\dagger l} e^{-2 \gamma t a^{\dagger} a}|n\rangle\langle n| a^{l} \\
= & \sum_{l=0}^{\infty} \frac{\left(1-e^{-2 t \gamma}\right)^{l}}{l !}: \exp \left[\left(e^{-2 \gamma t}-1\right) a^{\dagger} a\right]\left(a^{\dagger} a\right)^{l}:=1,
\end{aligned}
$$

from which one can see that the normalization still holds, i.e., they are trace-preserving in a general sense, so $M_{m, n, l}$ and $\mathcal{M}_{m, n, l}^{\dagger}$ may be named the generalized Kraus operators.

\section{Reduction of $\rho(t)$ in some special cases}

In general, Eq. (25) indicates that the Kerr medium causes phase diffusion while the field in cavity is in amplitude-damping. In Eq. (25), when the decoherent time $t \rightarrow \infty$, the main contribution comes from the $m=n=0$ term, in this case $\Lambda \rightarrow 1$, then

$$
\rho(t \rightarrow \infty) \rightarrow \sum_{l=0}^{\infty}\left\langle l\left|\rho_{0}\right| l\right\rangle|0\rangle\langle 0|=| 0\rangle\langle 0|
$$

since $\operatorname{Tr} \rho_{0}=1$, which shows that the quantum system reduces to the vacuum state after a long decoherence interaction, as expected.

In particular, when $\chi=0$, Eq.(25) becomes

$$
\begin{aligned}
\rho(t) & =\sum_{m, n, l=0}^{\infty} \sqrt{\frac{(n+l) !(m+l) !}{n ! m !}} \frac{\left(1-e^{-2 \gamma t}\right)^{l}}{l !} e^{-\gamma t(m+n)} \rho_{0, m+l, n+l}|m\rangle\langle n| \\
& =\sum_{m, n, l=0}^{\infty} \frac{\left(1-e^{-2 \gamma t}\right)^{l}}{l !} e^{-\gamma t a^{\dagger} a}|m\rangle\left\langle m\left|a^{l} \rho_{0} a^{\dagger l}\right| n\right\rangle\langle n| e^{-\gamma t a^{\dagger} a} \\
& =\sum_{l=0}^{\infty} \frac{\left(1-e^{-2 \gamma t}\right)^{l}}{l !} e^{-\gamma t a^{\dagger} a} a^{l} \rho_{0} a^{\dagger l} e^{-\gamma t a^{\dagger} a},
\end{aligned}
$$

which corresponds to the amplitude decaying mode, coinciding with the result in Ref. [6]. While for $\gamma=0$, Eq.(25) reduces to

$$
\rho(t)=\sum_{m, n=0}^{\infty} e^{-i \chi t\left(m^{2}-n^{2}\right)}|m\rangle\left\langle m\left|\rho_{0}\right| n\right\rangle\langle n|=e^{-i \chi\left(a^{\dagger} a\right)^{2} t} \rho_{0} e^{i \chi\left(a^{\dagger} a\right)^{2} t},
$$

which implies a process of phase diffusion, for $\rho_{0}=|n\rangle\langle n|$, then

$$
\rho(t)=e^{-i \chi\left(a^{\dagger} a\right)^{2} t}|n\rangle\left\langle n\left|e^{i \chi\left(a^{\dagger} a\right)^{2} t}=\right| n\right\rangle\langle n|,
$$


which shows neither decay nor phase diffusion happens in the Kerr medium.

In summary, we have demonstrated through the above example that the nonlinear Hamiltonian in master equation may demand us to modify Eq. (1), the general expression of superoperator.

Note added. Recently, we were made aware of Refs.[18] which deal with the Kerr medium using thermo field dynamic theory.

Acknowledgement Work supported by the National Natural Science Foundation of China under Grant 10775097 and 10874174. L.Y. Hu acknowledges Professor V. Srinivasan for his kind attention about Refs.[18].

\section{References}

[1] W. H. Louisell, Quantum Statistical Properties of Radiation (Wiley, New York, 1973).

[2] H. J. Carmichael, Statistical Methods in Quantum Optics 1: Master Equations and FokkerPlanck Equations, Springer-Verlag, Berlin, 1999; H. J. Carmichael, Statistical Methods in Quantum Optics 2: Non-Classical Fields, (Springer-Verlag, Berlin, 2008).

[3] J. Preskill, Lecture Notes for Physics 229, Quantum Information and Computation. CIT, 1998.

[4] K. Kraus, States, Effects, and Operations: Fundamental Notions of Quantum Theroy. Lecture Notes in Physics, Vol. 190. (Springer-Verlag, Berlin, 1983).

[5] K. E. Hellwig and K. Kraus, Pure operations and measurements. Commun. Math. Phys. 11, 214 (1969); Pure operations and measurements II. Commun. Math. Phys. 16, 142 (1970).

[6] H. Y. Fan and L. Y. Hu, Opt. Commun. 281 (2008) 5571; H. Y. Fan and L. Y. Hu, Opt. Commun. 2008 to appear

[7] for a recent review, see H. Y. Fan and L. Y. Hu, Mod, Phys. Lett. B 22 (2008) 2435

[8] Memorial Issue for H. Umezawa, Int. J. Mod. Phys. B 10, (1996) 1695 memorial issue and references therein.

[9] H. Umezawa, Advanced Field Theory - Micro, Macro, and Thermal Physics (AIP 1993)

[10] Y. Takahashi and H. Umezawa, Collecive Phenomena 2, (1975) 55.

[11] Hong-yi Fan and Yue Fan, Phys. Lett. A 246, (1998) 242; ibid, 282, (2001) 269.

[12] Hong-yi Fan, Hai-liang Lu and Yue Fan, Ann. Phys. 321, (2006) 480.

[13] A. Wünsche, J. Opt. B: Quantum Semiclass. Opt. 1, (1999) R11.

[14] G. J. Milburn and C. A. Kolmes, Phys. Rev. Lett. 56, (1986) 2237; D. J. Daniel and G. J. Milburn, Phys. Rev. A 39, (1989) 4628

[15] S. Chaturvedi, V. Srinivasan and G. S. Agarwal, J. Phys. A. Math. Gen 32 (1999) 1909.

[16] Wolfgang P. Schleich, Quantum Optics in Phase Space (Wiley-VCH, Birlin, 2001).

[17] Hong-yi Fan, Representation and Transformation Theory in Quantum Mechanics, (Shanghai Scientific \& Technical, Shanghai, 1997) (in Chinese).

[18] S. Chaturvedi and V. Srinivasan J. Mod. Opt. 38, (1991) 777; S. Chaturvedi and V. Srinivasan Phys. Rev. A 43, (1991) 4054. 